\title{
Antimicrobial susceptibility of organisms causing community-acquired urinary tract infections in Gauteng Province, South Africa
}

\author{
D A Lewis, ${ }^{1,2,3}$ FRCP (UK), PhD; L Y E Gumede, ${ }^{1}$ N Dip M Tech; L A van der Hoven, ${ }^{4}$ MB ChB; G N de Gita, ${ }^{1}$ MSc (Stats); E J E de Kock, \\ Dip Pharm; T de Lange ${ }^{6}$; V Maseko, ${ }^{1}$ B Tech; V Kekana, ${ }^{1}$ RN (PHC); F P Smuts, ${ }^{4}$ BSc (Hons); O Perovic, ${ }^{7,8}$ FCPath (Micro) SA, MMed
}

\author{
${ }^{1}$ Centre for HIV and Sexually Transmitted Infections, National Institute for Communicable Diseases, National Health Laboratory Service, \\ Johannesburg, South Africa \\ ${ }^{2}$ Department of Internal Medicine, Faculty of Health Sciences, University of the Witwatersrand, Johannesburg, South Africa \\ ${ }^{3}$ Division of Medical Microbiology, University of Cape Town, Cape Town, South Africa \\ ${ }^{4}$ Merck, Johannesburg, South Africa \\ Retrasol Research and Training Solutions, Pretoria, South Africa \\ ${ }^{6}$ DamaneX, Centurion, South Africa \\ ${ }^{7}$ Centre for Opportunistic, Tropical and Hospital Infections, National Institute for Communicable Diseases, National Health Laboratory Service, \\ Johannesburg, South Africa \\ ${ }^{8}$ Department of Clinical Microbiology and Infectious Diseases, Faculty of Health Sciences, University of the Witwatersrand, Johannesburg, South \\ Africa
}

Corresponding author:D A Lewis (davidl@nicd.ac.za)

\begin{abstract}
Background. Patients with community-acquired urinary tract infections (UTIs) frequently present to healthcare facilities in South Africa (SA). Aim. To provide information on UTI aetiology and antimicrobial susceptibility of pathogens.

Methods. We recruited women with UTI-related symptoms, who tested positive for $\geq 2$ urine dipstick criteria (proteinuria, blood, leucocytes or nitrites) at 1 public and 5 private primary healthcare facilities in 2011. Demographic and clinical data were recorded and mid-stream urine (MSU) specimens were cultured. UTI pathogens were Gram-stained and identified to species level. Etest-based antimicrobial susceptibility testing was performed for amoxicillin/clavulanic acid, cefixime, cefuroxime, ciprofloxacin, fosfomycin, levofloxacin, nitrofurantoin, norfloxacin and trimethoprim/sulphamethoxazole.

Results. Of the 460 women recruited, 425 MSU samples were processed and 204 UTI pathogens were identified in 201 samples. Most pathogens were Gram-negative bacilli (GNB) (182; 89.2\%) and 22 (10.8\%) were Gram-positive cocci (GPC). Escherichia coli was the most frequent GNB (160; 79.6\%), while Enterococcus faecalis was the predominant GPC $(8 ; 4.0 \%)$. The UTI pathogens had similar susceptibility profiles for fosfomycin (95.5\%; 95\% confidence interval (CI) 92.6 - 98.4), the 3 fluoroquinolones ( $94.1 \%$; $95 \%$ CI 90.8 - 97.4), nitrofurantoin (91.7\%; 95\% CI 87.8 - 95.6), cefuroxime (90.1\%; 95\% CI 86.0 - 94.3) and cefixime (88.2\%; 95\% CI 83.7 - 92.6). UTI pathogens were less susceptible to amoxicillin/clavulanic acid ( $82.8 \%$; $95 \%$ CI 77.5 - 88.0) when compared with fluoroquinolones and fosfomycin. Trimethoprim/ sulphamethoxazole was the least efficacious antimicrobial agent (44.3\% susceptible; 95\% CI 37.4 - 51.2).

Conclusion. This study provides relevant data for the empirical treatment of community-acquired UTIs in SA.

S Afr Med J 2013;103(6):377-381. DOI:10.7196/SAMJ.6722
\end{abstract}

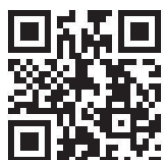

Community-acquired urinary tract infection (UTI) is a common medical condition affecting up to $20 \%$ of women sometime in their lifetime. These infections are usually diagnosed in previously healthy women with no underlying disease or anatomical anomalies. ${ }^{[1]}$ Several risk factors have been described in the literature: (i) frequent sexual intercourse; (ii) lack of urination after sexual intercourse; (iii) a new sexual partner in the past year; (iv) use of a diaphragm or spermicide; $(v)$ a history of recurrent UTI, and $(v i)$ the occurrence of UTI in a first-degree female relative. ${ }^{[1,2]}$

Escherichia coli is the most common community-acquired UTI pathogen and is responsible for $75-95 \%$ of cases. ${ }^{[1]}$ The remaining infections are usually caused by Gram-negative Enterobacteriaceae or Gram-positive cocci (GPC). ${ }^{[1]}$ The extensive use of antimicrobial agents to treat community-acquired UTIs has resulted in the emergence of antimicrobial resistance among some of these bacterial species, particularly in developing countries. ${ }^{[3,4]}$ Of current concern is the increased prevalence of community-acquired extended-spectrum $\beta$-lactamase (ESBL)-producing organisms. ${ }^{[5]}$

Since recent aetiological and antimicrobial susceptibility data relating to community-acquired UTIs within South Africa (SA) are scarce, we undertook such a survey among women attending public and private healthcare facilities in Gauteng Province.

\section{Methods \\ Study design}

We conducted a multi-centre cross-sectional study to determine the prevalence and antimicrobial-resistance profile of bacteria causing community-acquired UTIs in women. We enrolled sufficient symptomatic women to achieve a recruitment target of 200 women with microbiologically confirmed UTIs. The study took place in Gauteng Province at 1 public primary healthcare site and 5 private practitioners' clinics between March and November 2011. Participation took place on the day of study enrolment and no 
follow-up visits were required. The study was approved by the Human Research Ethics Committee (Medical) of the University of the Witwatersrand (M101119) and Pharma Ethics.

\section{Participant screening and enrolment}

The women, aged $18-80$ years were screened by urinalysis. Eligibility criteria included: (i) presenting with UTI symptoms, and (ii) the provision of informed written consent. Exclusion criteria included women with a history of current renal disease or known urinary tract abnormalities, as well as recent in-patient hospitalisation or the use of antimicrobial agents within the previous month. We enrolled women if their mid-stream urine (MSU) was positive for $\geq 2 / 4$ criteria (protein, blood, leucocytes or nitrites); using either Quick Profile urine reagent strips (LumiQuick Diagnostics, USA) or the Uryxxon Relax automated urinalysis system (Macherey-Nagel, Germany).

\section{Treatment of participants}

We treated women attending the public clinic according to Essential Drugs List (EDL) recommendations with either a single $500 \mathrm{mg}$ dose of ciprofloxacin (for non-pregnant participants) or with a 1-week course of $250 \mathrm{mg}$ amoxicillin/125 mg clavulanic acid given 8-hourly (for pregnant participants). ${ }^{[6]}$ We examined women attending private clinics and treated them according to their practitioner's antibiotic preference. We recorded the antimicrobial agents used to treat the presumptive UTIs on a laboratory tracker form that accompanied the MSU sample to the laboratory. Clinicians received antimicrobial susceptibility results only for those cases in which a urinary pathogen was isolated and determined to be resistant to the dispensed antimicrobial agent.

\section{Bacterial isolation, species identification and laboratory reporting criteria}

MSU specimens were sent in a cooler box to the National Institute for Communicable Diseases (NICD). We discarded MSU specimens that were $>24$ hours old on arrival at the NICD. Urine specimens were processed according to a routine standard operative procedure by using a $1 \mu \mathrm{l}$ loop to inoculate both $5 \%$ blood and MacConkey agar plates (Diagnostic Media Products, SA). Inoculated plates were incubated for 18 hours at $35-37^{\circ} \mathrm{C}$ and then inspected for bacterial growth. We regarded the presence of $\geq 10$ identical colonial morphotypes $\left(\geq 10^{4}\right.$ colony-forming units (CFU)/ml) as microbiological evidence of a UTI. If $<10$ bacterial colonies were present, the urine was reported to have 'no significant growth'. If 2 different bacterial colony types were present in equal numbers, both were purified for further laboratory work. If $\geq 3$ bacterial colony types were present, then the urine was reported as 'mixed growth' and the plates were discarded. The predominant colonies were re-cultured on $5 \%$ blood agar prior to identification and antimicrobial susceptibility testing. Bacterial colonies were initially classified by Gram-stain morphology and subsequently definitively identified using the VITEK 2 system (bioMérieux SA, France). Bacterial strains were archived at $-70^{\circ} \mathrm{C}$ in Microbank storage vials (Pro-Lab Diagnostics, Canada) for the study duration.

\section{Antimicrobial susceptibility testing}

Bacterial species were first suspended in $2 \mathrm{ml} 0.9 \%$ sterile saline to turbidity equivalent to a $0.5 \mathrm{McFarland}$ standard $\left(10^{8} \mathrm{CFU} / \mathrm{ml}\right)$. A cotton-tipped swab was used to inoculate appropriate media (Diagnostics Media Products, SA) with the bacterial suspension. As recommended by the Clinical Laboratory Standards Institute (CLSI), we used Mueller-Hinton agar to perform antimicrobial susceptibility testing for either Gram-negative bacilli (GNB) or GPC in clusters, while Mueller-Hinton agar with 5\% sheep's blood was used for GPC in chains. ${ }^{[7]}$ Susceptibility to the following antimicrobial agents was determined immediately after pathogen identification using Etest strips (AB Biodisk, Sweden): cefixime, cefuroxime, ciprofloxacin, norfloxacin, levofloxacin, amoxicillin/ clavulanic acid and trimethoprim/sulphamethoxazole. After recruitment had been completed, we performed additional Etestbased minimum inhibitory concentration (MIC) assays for 2 further antimicrobial agents, fosfomycin and nitrofurantoin, using bacterial isolates cultured from $-70^{\circ} \mathrm{C}$ stock vials in the same manner as described above. For fosfomycin, glucose-6-phosphate $(25 \mathrm{mg} / \mathrm{l})$ was incorporated into the Mueller-Hinton agar, as recommended by CLSI guidelines. ${ }^{[7]}$ We used 3 bacterial strains as controls for antimicrobial susceptibility testing: E. coli (American Type Culture Collection (ATCC) 25922); Staphylococcus aureus (ATCC 25923); Streptococcus pneumoniae (ATCC 49619). After placing Etest strips on the inoculated agar surface, we incubated plates for 24 hours before reading the MIC of each antimicrobial agent for each isolate, according to manufacturer's instructions.

Wherever possible, we interpreted MICs according to CLSI breakpoint criteria. ${ }^{[7]}$ As surrogate antibiotics for cefixime we used the CLSI breakpoints for the parenteral third-generation cephalosporins, ceftizoxime and ceftriaxone, for GPC in clusters and for $\beta$-haemolytic streptococci, respectively. Since enterococci are inherently nonsusceptible to cephalosporins, they were reported as such, regardless of MIC value. For $\beta$-haemolytic streptococci we reported: (i) susceptibility to amoxicillin/clavulanic acid using surrogate CLSI breakpoints for ampicillin; (ii) susceptibility to cefuroxime using surrogate CLSI breakpoints for ceftriaxone; and (iii) susceptibility to trimethoprim/ sulphamethoxazole using surrogate CLSI breakpoints for S. pneumoniae. When testing fosfomycin against GPC, we used the antimicrobial susceptibility breakpoints of the British Society for Antimicrobial Chemotherapy (BSAC) for staphylococci (version 10.2, May 2011) for both GPC in clusters and $\beta$-haemolytic streptococci. ${ }^{[8]}$ We also used the BSAC breakpoints to determine susceptibility of $\beta$-haemolytic streptococci to nitrofurantoin.

\section{Data analysis}

Data were entered into a Microsoft Access database and analysed using Microsoft Excel. We determined the $\mathrm{MIC}_{50}$ and $\mathrm{MIC}_{90}$ values for each antimicrobial agent and investigated associations between variables of interest using the chi-squared test. All statistical analyses were conducted using STATA version 10.1 (StataCorp, USA).

\section{Results}

\section{Participant recruitment and characteristics}

Overall, 460 participants were recruited at the 1 public $(n=230)$ and the 5 private practice facilities $(n=230)$. The mean participant age was lower at the public sector facility ( $31.0 \mathrm{v} .34 .7$ years). The age range of recruited participants did not differ markedly by facility type (18 - 74 v. $18-79$ years in public and private sector, respectively). Almost all participants attending the public facility were black Africans (229; $99.6 \%$ ), while the racial distribution was more varied at the private practice facilities (139 white (60.4\%); 56 black African (24.3\%); 21 Indian (9.1\%); 10 coloured (4.3\%); 4 unknown (1.7\%)).

\section{Urinalysis and clinical symptoms}

The frequency distribution of detected urine dipstick abnormalities and UTI-related clinical symptoms is shown in Table 1. Isolation of a UTI pathogen was significantly associated with proteinuria $(p=0.008)$, the presence of nitrites $(p<0.001)$, dysuria $(p<0.001)$, urgency $(p<0.001)$, frequency $(p<0.001)$ and strong-smelling urine $(p<0.001)$. In contrast, failure to isolate a bacterial pathogen was 
Table 1. Frequency of positive urine-dipstick screening criteria and clinical symptoms reported $(N=425)$

\begin{tabular}{ll}
\hline Diagnostic criteria & $\begin{array}{l}\text { Patients } \\
\boldsymbol{n}(\%)\end{array}$ \\
\hline Urinalysis & $321(75.5)$ \\
Protein & $314(73.9)$ \\
Blood & $228(53.7)$ \\
Leucocytes & $94(22.1)$ \\
Nitrites & \\
Clinical symptoms & $336(79.1)$ \\
Lower abdominal pain & $324(76.2)$ \\
Dysuria & $220(51.8)$ \\
Frequency of micturition & $160(37.7)$ \\
Discoloured urine & $148(34.8)$ \\
Cloudy urine & $138(32.5)$ \\
Urgency & $124(29.2)$ \\
Strong smell to urine & $69(12.2)$ \\
Loin or upper back pain & $45(10.6)$ \\
Nausea and/or vomiting & $28(6.6)$ \\
Chills or fevers &
\end{tabular}

Table 2. Frequency of the community-acquired UTI pathogens $(N=204)$ isolated from patients $(N=201)$ by Gram stain morphotype and facility type

\begin{tabular}{|c|c|c|c|}
\hline \multirow[b]{2}{*}{ UTI pathogens* } & \multicolumn{3}{|c|}{ Patients by facility type } \\
\hline & $\begin{array}{l}\text { Public patients } \\
(N=107) \\
n(\%)\end{array}$ & $\begin{array}{l}\text { Private patients } \\
(N=94) \\
n(\%)\end{array}$ & $\begin{array}{l}\text { All patients } \\
(N=201) \\
n(\%)\end{array}$ \\
\hline \multicolumn{4}{|l|}{ GNB $(n=182)$} \\
\hline E. coli & $83(77.6)$ & $77(81.9)$ & $160(79.6)$ \\
\hline Proteus mirabilis & $6(5.6)$ & $4(4.3)$ & $10(5.0)$ \\
\hline Citrobacter koseri & - & $1(1.1)$ & $1(0.5)$ \\
\hline Klebsiella pneumoniae & $4(3.7)$ & $1(1.1)$ & $5(2.5)$ \\
\hline Leclercia adecarboxylata & - & $1(1.1)$ & $1(0.5)$ \\
\hline Enterobacter aerogenes & $3(2.8)$ & - & $3(1.5)$ \\
\hline Salmonella spp. & $1(0.9)$ & - & $1(0.5)$ \\
\hline Unidentified $^{\dagger}$ & $1(0.9)$ & - & $1(0.5)$ \\
\hline \multicolumn{4}{|l|}{$\operatorname{GPC}(n=22)$} \\
\hline E. faecalis & $1(0.9)$ & $7(7.4)$ & $8(4.0)$ \\
\hline Staphylococcus saprophyticus & $3(2.8)$ & $2(2.1)$ & $5(2.5)$ \\
\hline Streptococcus agalactiae & $5(4.7)$ & $2(2.1)$ & $7(3.5)$ \\
\hline Staphylococcus haemolyticus & $1(0.9)$ & $1(1.1)$ & $2(1.0)$ \\
\hline
\end{tabular}

significantly associated with discoloured urine $(p=0.037)$ and pregnancy $(p<0.001)$. There were no other significant associations between UTI pathogen isolation and the other diagnostic criteria listed in Table 1. prescribed to participants in accordance with the EDL guideline. The study nurse reported drug stock-out at the facility's pharmacy on some days during the study; accordingly, other drugs had to be used to treat presumptive UTIs in the remaining 19 $(8.3 \%)$ cases. Antimicrobial prescribing was more varied at the private practice facilities for the $151(65.7 \%)$ participants for whom data were recorded. Ciprofloxacin was the most frequent therapeutic choice (76; $50.3 \%)$, followed by fosfomycin $(27 ; 17.9 \%)$, amoxicillin/clavulanic acid $(23 ; 15.2 \%)$, cefuroxime $(10 ; 6.6 \%)$, cefpodoxime $(7$; $4.6 \%)$, levofloxacin $(6 ; 4.0 \%)$ and amoxicillin (2; $1.3 \%)$.

\section{Laboratory culture and identification of UTI pathogens}

Within the laboratory we processed 425 (92.4\%) MSU samples; 35 (7.6\%) specimens were discarded on the basis of being $>24$ hours old. We isolated bacteria in significant numbers in 203 (47.8\%) participants; most were single organisms $(200 ; 98.5 \%)$ while 3 participants (1.5\%) had 2 different bacterial species identified. Culture of the MSU samples of 2 patients grew Gram-positive bacteria, Kocuria kristinae and Pediococcus pentosaceus, that we regarded as skin and vaginal commensals rather than UTI pathogens. Accordingly, we did not include these 2 isolates in the antimicrobial susceptibility analyses. Plates with 'no significant growth', 'no growth' or 'mixed growth' were reported for $138(32.2 \%), 84(19.6 \%)$ and $0(0.0 \%)$ participants, respectively.

After excluding the 2 commensal isolates described above, we identified a total of 204 UTI pathogens in 201 participants (Table 2). In terms of Gram-stain morphotype, 182 (89.2\%) were GNB and 22 (10.8\%) were GPC (Table 2). Self-reported pregnancy status was available for most of these participants (199/201, 99.0\%). The prevalence of GPC was significantly higher among pregnant than non-pregnant women $(5 / 19(26.3 \%) \mathrm{v}$. $17 / 180$ (9.4\%), respectively; $p=0.026)$. There were no significant associations between the detection of a UTI pathogen or the Gram stain morphotype and either the participant's racial group $(p=0.448$ and $p=0.734$, respectively) or the facility type ( $p=0.944$ and $p=0.456$, respectively). Identification of the UTI pathogens-to-species level, as determined by the VITEK 2 system, demonstrated that E. coli accounted for just over three-quarters of all UTI pathogens isolated $(160 ; 79.6 \%)$, while $E$. faecalis was the most frequent Gram-positive organism detected $(8 ; 4.0 \%)$ (Table 2). There was a 
Table 3. Antimicrobial susceptibility profiles for the antimicrobial agents tested against UTI pathogens by Gram-stain morphotype

\begin{tabular}{|c|c|c|c|c|c|c|c|c|}
\hline \multirow{3}{*}{$\begin{array}{l}\text { Antimicrobial agent } \\
(N=9)\end{array}$} & \multicolumn{4}{|c|}{$\begin{array}{c}\text { GNB } \\
(N=181)^{*}\end{array}$} & \multicolumn{2}{|c|}{$\begin{array}{l}\mathrm{GPC}^{\dagger} \\
(N=22)^{\ddagger}\end{array}$} & \multicolumn{2}{|c|}{$\begin{array}{l}\text { All } \\
(N=203)\end{array}$} \\
\hline & \multicolumn{2}{|r|}{ Susceptibility } & \multirow{2}{*}{$\begin{array}{l}\mathrm{MIC}_{50} \\
(\mathrm{mg} / \mathrm{l})\end{array}$} & \multirow{2}{*}{$\begin{array}{r}\mathrm{MIC}_{90} \\
(\mathrm{mg} / \mathrm{l})\end{array}$} & \multicolumn{2}{|r|}{ Susceptibility } & \multicolumn{2}{|r|}{ Susceptibility } \\
\hline & $n$ & $\%(95 \% \mathrm{CI})$ & & & $n$ & $\%(95 \% \mathrm{CI})$ & $n$ & $\%(95 \% \mathrm{CI})$ \\
\hline Ciprofloxacin & 170 & $93.9(90.4-97.4)$ & 0.012 & 0.19 & 21 & $95.5(86.0-100.0)$ & 191 & $94.1(90.8-97.4)$ \\
\hline Levofloxacin & 170 & $93.9(90.4-97.4)$ & 0.023 & 0.38 & 21 & $95.5(86.0-100.0)$ & 191 & $94.1(90.8-97.4)$ \\
\hline Norfloxacin & 170 & $93.9(90.4-97.4)$ & 0.064 & 0.75 & 21 & $95.5(86.0-100.0)$ & 191 & $94.1(90.8-97.4)$ \\
\hline Cefixime & 172 & $95.0(91.8-98.2)$ & 0.25 & 0.75 & 7 & $31.8(10.7-53.0)$ & 179 & $88.2(83.7-92.6)$ \\
\hline Cefuroxime & 169 & $93.4(89.7-97.0)$ & 3 & 4 & 14 & $63.6(41.8-85.5)$ & 183 & $90.1(86.0-94.3)$ \\
\hline Fosfomycin ${ }^{5}$ & 176 & $98.3(96.4-100.0)$ & 0.5 & 4 & 16 & $72.7(52.5-92.9)$ & 195 & $95.5(92.6-98.4)$ \\
\hline Nitrofurantoin & 155 & $90.6(86.2-95.1)$ & 12 & 32 & 22 & $100.0(100.0-100.0)$ & 177 & $91.7(87.8-95.6)$ \\
\hline Amoxicillin/clavulanic acid & 146 & $80.7(74.9-86.5)$ & 6 & 16 & 22 & $100.0(100.0-100.0)$ & 168 & $82.8(77.5-88.0)$ \\
\hline Trimethoprim/sulphamethoxazole & 75 & $41.4(34.2-48.7)$ & $>32$ & $>32$ & 15 & $68.2(47.0-89.3)$ & 90 & $44.3(37.4-51.2)$ \\
\hline 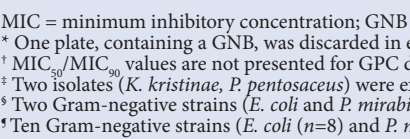 & $\begin{array}{l}\text { he heter } \\
\text { from } t \\
\text { s unava } \\
(n=2))\end{array}$ & $\begin{array}{l}\text { ive bacilli; GPC = Gram-p } \\
\text { ecies identification and an } \\
\text { rogeneous nature of this gr } \\
\text { he antimicrobial susceptib } \\
\text { tilable for fosfomycin susce } \\
\text { were unavailable for nitro }\end{array}$ & $\begin{array}{l}\text { up. } \\
\text { ty analysis } \\
\text { tibility tes }\end{array}$ & they were & & e commensal contaminants & th & ue UTI pathogens. \\
\hline
\end{tabular}

significantly higher prevalence of E. faecalis isolation in MSU samples collected in the private facilities than in the public facility $(7 / 94(7.4 \%)$ v. $1 / 107(0.9 \%)$, respectively; $p=0.018)$.

\section{Antimicrobial susceptibility}

In error, we discarded 1 UTI pathogen, a GNB, prior to antimicrobial susceptibility testing. Table 3 shows the susceptibility of the remaining 203 UTI pathogens to the 9 antimicrobial agents tested, as well as the GNB-related $\mathrm{MIC}_{50}$ and $\mathrm{MIC}_{90}$ values for each antimicrobial agent. A few of the bacterial isolates were not available for post-recruitment susceptibility testing with fosfomycin (2 isolates) and nitrofurantoin (10 isolates). Overall, the UTI pathogens were most susceptible to fosfomycin (95.5\%; $95 \%$ confidence interval (CI) 92.6 - 98.4), the 3 fluoroquinolones (94.1\%; 95\% CI 90.8 - 97.4), nitrofurantoin (91.7\%; 95\% CI 87.8 - 95.6), cefuroxime (90.1\%; 95\% CI 86.0 - 94.3) and cefixime (88.2\%; 95\% CI 83.7 -92.6). The performance of these 7 antimicrobial agents was regarded as similar based on overlapping $95 \%$ CIs. Although the UTI pathogens were less susceptible to amoxicillin/clavulanic acid $(82.8 \%$; 95\% CI 77.5 - 88.0) than to fluoroquinolones and fosfomycin; amoxicillin/clavulanic acid had a similar susceptibility profile to cefuroxime, cefixime and nitrofurantoin. Trimethoprim/ sulphamethoxazole was the least efficacious antimicrobial agent (44.3\% susceptibility; 95\% CI 37.4 - 51.2).

\section{Discussion}

This study describes the aetiology of community-acquired UTIs in women presenting to public and private facilities in Gauteng Province. In keeping with the existing literature, most bacterial isolates were GNB including E. coli, which accounted for $80 \%$ of bacterial isolates. ${ }^{[1]}$ The UTI pathogens tested were most susceptible to fluoroquinolones, cephalosporins, fosfomycin and nitrofurantoin. While these pathogens were less susceptible to amoxicillin/clavulanic acid than to fluoroquinolones and fosfomycin, amoxicillin/clavulanic acid had a similar susceptibility profile to both cephalosporins and nitrofurantoin. In contrast, $>50 \%$ of the UTI pathogens tested were susceptible to trimethoprim/sulphamethoxazole. In terms of directed therapy based on known Gram-stain morphotypes, our data suggest that fosfomycin, fluoroquinolones and cephalosporins would be good choices for GNB infections while GPC infections may be treated best with amoxicillin/clavulanic acid, nitrofurantoin or fluoroquinolones.

Community-acquired UTIs continue to represent a financial burden for many countries and short-course treatments are favoured, where possible, as resistance develops less commonly. ${ }^{[9]}$ As antimicrobial resistance escalates, the cost of managing such infections will increase as more expensive antimicrobial agents are required to ensure microbiological cure. Within SA, most community-acquired UTIs are treated empirically without the need for laboratory investigation and susceptibility testing of isolated UTI pathogens. The lack of community-derived microbiological data presents challenges to those responsible for recommending firstline UTI therapy/ies. The choice of first-line antimicrobial agents is determined by a number of factors, including in vitro susceptibility, side-effect profile, patient allergy, patient's compliance profile, financial cost to the healthcare system or the patient, therapeutic efficacy, and the agent's propensity for producing an ecological adverse effect, i.e. the development of further bacterial antimicrobial resistance over time due to selection pressure. ${ }^{[10]}$

Maartens and Oliver ${ }^{[11]}$ undertook a similar microbiological study of community-acquired UTI pathogens to determine the prevalence of antimicrobial resistance in Cape Town, SA. Their study, performed over a decade ago with different methodology, reported an almost identical prevalence of resistance to trimethoprim/sulphamethoxazole (47.3\%), but a relatively low level of resistance to ciprofloxacin $(2.8 \%)$. Our data confirm the rising prevalence of fluoroquinolone resistance among community-acquired UTI pathogens in SA. ${ }^{[12]}$ The Infectious Diseases Society of America (IDSA) recently published guidelines that recommend a $10 \%$ resistance threshold for the treatment of community-acquired UTIs with fluoroquinolones; above this threshold, fluoroquinolones should not be used as empirical treatment. ${ }^{[13]}$ Fluoroquinolones offer broad-spectrum UTI pathogen cover with few side-effects and are suitable for the treatment of complicated UTI and pyelonephritis; however, their main disadvantages are that they are contraindicated in pregnancy and they have a high propensity for ecological adverse effects. ${ }^{[1]}$ 
Fosfomycin has a broad antibacterial spectrum and inhibits phosphoenolpyruvate transferase, the first enzyme involved in peptidoglycan synthesis. ${ }^{[14]}$ Like nitrofurantoin, fosfomycin is only suitable to treat lower UTIs. Administered as a single oral dose of $3 \mathrm{~g}$ fosfomycin-trometamol, it has few side-effects and is safe in pregnancy, it does not exhibit cross-resistance and it is useful to treat multidrug-resistant UTI pathogens, including ESBL-producing Enterobacteriaceae and vancomycin-resistant enterococci. ${ }^{[14-16]} \mathrm{A}$ major disadvantage of fosfomycin is its relatively high cost in comparison with the other antimicrobial agents currently in use for the treatment of community-acquired UTIs. In terms of clinical efficacy, there are some concerns about unacceptably high prevalence of bacterial persistence. ${ }^{[17]}$ Although fosfomycin resistance infrequently occurs as a result of chromosomal mutations, resistant bacteria exhibit a reduced rate of growth and appear less virulent in terms of their ability to adhere to uro-epithelial cells in vitro. ${ }^{[18]}$

Our data suggest that cephalosporins are best suited as a treatment option for directed therapy for confirmed Gram-negative UTIs. This differential susceptibility of cephalosporins on the basis of Gramstain morphotype has been described previously in SA. ${ }^{[10]}$ Although cefixime, an oral third-generation cephalosporin, may be used as a single $400 \mathrm{mg}$ dose for the treatment of UTI, it is presently only available for the treatment of gonorrhoea in the SA public sector. Oral cephalosporins have the advantage of being safe to prescribe in pregnancy and induce few side-effects.

Nitrofurantoin monohydrate macrocrystalline formulation, given orally as a 5-day course, is well tolerated, has good efficacy and a low propensity for ecologically adverse events. Nitrofurantoin is recommended as one of several first-line agents in the IDSA 2010 guidelines and, within SA, it is also recommended for short-term prophylaxis in women with recurrent UTIs. Nitrofurantoin may be either bacterio-static or -cidal in action, depending on the concentration and susceptibility of the infecting organism, and exerts its effects by inhibiting DNA, RNA, protein and cell wall synthesis. Side-effects are generally minor, such as nausea and urine discolouration, but haemolytic anaemia is a risk in those patients with glucose-6-phosphate dehydrogenase deficiency, and pulmonary fibrosis may occur with long-term use.

A strength of our study is that it was undertaken at both public and private sector healthcare facilities in Gauteng Province; however, the findings may not be nationally representative. Additionally, to enhance organism yield, we selected participants after initial screening by urinalysis, which could present a bias in pathogen selection. For some of the antibiotics it was difficult to determine the category of antimicrobial susceptibility due to the absence of appropriate CLSI or BSAC MIC breakpoints. A further limitation is that we measured in vitro resistance rates; these data may overestimate clinically relevant resistance to the antimicrobial agents tested. Finally, although injectable gentamicin and ceftriaxone are recommended for the treatment of severe upper UTIs in the SA EDL, they were not tested in the current study.

In conclusion, our study provides valuable local data for empirical antimicrobial therapy for community-acquired UTIs. In view of rising antimicrobial resistance trends among UTI pathogens, we recommend that consideration be given to the establishment of a national sentinel surveillance system to monitor the antimicrobial susceptibility profiles of community-acquired UTI pathogens within
SA. Such a programme would generate the required evidence base to support antibiotic guideline and policy creation.

Acknowledgements. We thank Prof. H S Schoeman for the statistical study report he provided to Merck Serono at the end of the study and Dr A Brink for his insightful comments during protocol design and implementation of the study. We express our gratitude to the private practitioners who participated in the study: Drs W G Meyer and Y Steyn (Wierdamed); Drs C L Landman and M E Landman (Doctors CL \& ME Landman Inc.); Drs A S Kilian, J C van Staden, J J Nienaber and P K Williams-Scott (Kilian, Nienaber and Associates); Drs Y Le Roux, J J Le Roux and H J Sommerville (Healthworx Centurion); and Dr P Steytler (Unitas Hospital, Centurion). We thank Mr L Maluleke for permission to undertake the study at Alexandra Health Centre, and the clinical and laboratory staff of the Centre for HIV and STIs for their assistance.

Conflicts of interest. This study was sponsored by Merck, which is the manufacturer of cefixime. LvdH and FS were employed by Merck at the time of the study. EdK and TdL were contracted by Merck to assist with operational and data management aspects of the study, respectively. DL served as a voluntary member of the Merck Advisory Board during the time of the study.

\section{References}

1. Hooton TM. Clinical practice. Uncomplicated urinary tract infection. N Engl J Med 2012;366(11):10281037. [http://dx.doi.org/10.1056/NEJMcp1104429]

2. Jancel T, Dudas V. Management of uncomplicated urinary tract infections. West J Med 2002;176(1):5155. [http://dx.doi.org/10.1136/ewjm.176.1.51]
. Jancel T. Dudas V. Management of uncomplicated

55. [http://dx.doi.org/10.1136/ewjm.176.1.51]
Lester SC, del Pilar Pla M, Wang F, Perez Schael I, Jiang H, O’Brien TF. The carriage of Escherichic Lester SC, del Pilar Pla M, Wang F, Perez Schael I, Jiang H, O'Brien TF. The carriage of Escherichia
coli resistant to antimicrobial agents by healthy children in Boston, in Caracas, Venezuela, and in Qin coli resistant to antimicrobial agents by healthy children in Boston, in Caracas, Venezuela, and in Qin

4. Paterson DL. Resistance in gram-negative bacteria: Enterobacteriaceae. Am J Med 2006;119:S20-28. [http://dx.doi.org/10.1016/j.amjmed.2006.03.013]

5. Pitout JD, Laupland KB. Extended-spectrum beta-lactamase-producing Enterobacteriaceae: An emerging public-health concern. Lancet Infect Dis 2008;8(3):159-166. [http://dx.doi.org/10.1016/ S1473-3099(08)70041-0]

6. Essential Drugs Programme. Primary health care standard treatment guidelines and essential medicines list 2008. 4th ed. Pretoria: National Department of Health, 2008.

Clinical and Laboratory Standards Institute. Performance Standards for Antimicrobial Susceptibility Testing; Twenty-Second Informational Supplement M100-S22. Wayne, PA, USA: Clinical and Laboratory Standards Intite Jor uploads/2012/11/M100S22E.pdf (accessed 25 March 2013).

8. British Society for Antimicrobial Chemotherapy. BSAC methods for antimicrobial susceptibility Brish Socty for Animitrobility (accessed 25 March 2013)

9. Leibovici L, Wysenbeek AJ. Single-dose antibiotic treatment for symptomatic urinary tract infections in women: a meta-analysis of randomized trials. Q J Med 1991;78(285):43-57.

10. Bosch FJ, van Vuuren C, Joubert G. Antimicrobial resistance patterns in outpatient urinary trac infections - the constant need to revise prescribing habits. S Afr Med J 2011;101(5):328-331.

11. Maartens G, Oliver SP. Antibiotic resistance in community-acquired urinary tract infections. S Afr Med J 1994;84:600-602.

2. Habte TM, Dube S, Ismail N, Hoosen AA. Hospital and community isolates of uropathogens at a tertiary hospital in South Africa. S Afr Med J 2009;99(8):584-587.

13. Gupta K, Hooton TM, Naber KG, et al. International clinical practice guidelines for the treatment of acute uncomplicated cystitis and pyelonephritis in women: A 2010 update by the Infectious Diseases Society of America and the European Society for Microbiology and Infectious Diseases. Clin Infect Dis 2011;52(5):e103-120. [http://dx.doi.org/10.1093/cid/ciq257]

4. Raz R. Fosfomycin: an old-new antibiotic. Clin Microbiol Infect 2011;18(1):4-7. [http://dx.doi. Raz R. Fosfomycin: an old-new antibiti

arge Antimicrob Chemother 1999;43(2):211-217. [http://dx.doi.org/10.1093/jac/43.2.211]

Antimicrob Chemother 1999;43(2):211-217. [http://dx.doi.org/10.1093/jac/43.2.211]
16. Falagas ME, Kastoris AC, Kapaskelis AM, Karageorgopoulos DE. Fosfomycin for the treatment of multidrug-resistant, including extended-spectrum beta-lactamase producing, Enterobacteriaceae infections: a systematic review. Lancet Infect Dis 2010;10(1):43-50. [http://dx.doi.org/10.1016/S14733099(09)70325-1

7. Minassian MA, Lewis DA, Chattopadhyay D, Bovill B, Duckworth GJ, Williams JD. A comparison between single-dose fosfomycin trometamol (Monuril) and a 5-day course of trimethoprim in the treatment of uncomplicated lower urinary tract infection in women. Int J Antimicrob Agents 1998;10(1):39-47. [http://dx.doi.org/10.1016/S0924-8579(98)00021-1]

18. Marchese A, Gualco L, Debbia EA, Schito GC, Schito AM. In vitro activity of fosfomycin against gramnegative urinary pathogens and the biological cost of fosfomycin resistance. Int J Antimicrob Agents 2003:22:53-59. [http://dx.doi.org/10.1016/S0924-8579(03)00230-9] 